[In: Honig, M.-S. (Hrsg.) (2009): Ordnungen der Kindheit. Problemstellungen und Perspektiven der Forschung. Weinheim: Juventa, S. 79-102.]

\title{
Helga Kelle
}

\section{Kindliche Entwicklung und die Prävention von Entwicklungsstörungen. Die frühe Kindheit im Fokus der childhood studies}

In dem Beitrag wird die historische Entstehung des Paradigmas der (normalen) kindlichen Entwicklung, dessen gesellschaftliche und technologische Voraussetzungen sowie die Differenzierung von kinderwissenschaftlichen Disziplinen im 19. und 20. Jh. einleitend kurz nachgezeichnet. In einem zweiten Abschnitt wird die aktuelle Thematisierung bzw. Nichtthematisierung der „kindlichen Entwicklung“ im Rahmen der neueren Kindheitssoziologie diskutiert; die Autorin plädiert vor diesem Hintergrund dafür, die (frühe) „Entwicklungskindheit“ zu einem wissens- und praxissoziologisch grundierten Gebiet der Kindheitsforschung auszubauen und eine soziologische Re-Theoretisierung der kindlichen Entwicklung voranzutreiben. Dieses Plädoyer wird im folgenden Abschnitt daran plausibilisiert, dass die (normale) kindliche Entwicklung seit Ende des 20. Jh. in neuen Weisen unter gesellschaftlicher Beobachtung steht, und zwar insbesondere durch die Institutionalisierung von flächendeckenden, staatlich regulierten entwicklungsdiagnostischen Verfahren in der frühen Kindheit, die tendenziell auf eine Erfassung aller Kinder zielen. Im Fazit werden die Aufgaben, Gegenstandsebenen und methodologischen Herausforderungen einer (empirischen) Soziologie der kindlichen Entwicklung und Entwicklungsbeobachtung schließlich an einem eigenen Forschungsprojekt veranschaulicht.

Die „Entwicklungstatsache“ (Bernfeld 1925/1967) begründet wesentlich die soziale und kulturelle Differenz zwischen Kindern und Erwachsenen und legitimiert die generationale Ordnung von Gesellschaften. Dass Bernfeld von einer „Tatsache“ sprach, bezeugt seine Referenz an die anthropologischen Grundlagen von Erziehung: Kinder entwickeln sich in allen Kulturen, und in allen Kulturen bedürfen sie der Pflege und erzieherischen Assistenz, um sich überhaupt entwickeln zu können. Die Entwicklungstatsache als anthropologische Grundlage zu verstehen bedeutet allerdings noch nicht, dass man auch konkrete Phasen oder Stufen der Entwicklung von Kinderkörpern und -kompetenzen konzipieren muss. Die Ausbuchstabierung der Annahmen über die Prozesse der kindlichen Entwicklung ist vielmehr in hohem Maße historisch und kulturell relativ. Und auch die Grundannahme, dass Kindheit Entwicklungskindheit bedeutet, ist historisch eng verknüpft mit den Prozessen der gesellschaftlichen Institutionalisierung der Differenz zwischen Kindern und Erwachsenen - also mit der Etablierung der Kindheitsidee als solcher (vgl. Honig 1999). 


\section{Zur Entstehung des Paradigmas der (normalen) kindlichen Entwicklung}

Ideengeschichtlich betrachtet wird das Verb „entwickeln“ seit dem 18. Jahrhundert auf das Aufwachsen von Kindern bezogen. Rousseaus Konzeption der Unschuld und Unverbildetheit der kindlichen Natur hat maßgeblich dazu beigetragen, dass das Aufwachsen von Kindern als ein „Prozess der Vervollkommnung“ betrachtet werden konnte, als ein ,natürlicher' Prozess, „der sich zwar aus sich heraus vollzieht, aber stets als Forschritt konzipiert ist" (Tervooren 2008, S. 43). Deshalb spricht Honig (1999, S. 62) von einer „Teleologie“, die dem Entwicklungsgedanken immanent sei und es erlaube, „fast unbemerkt von einer Beschreibung kindlicher Entwicklung zu einer Normierung kindlicher Entwicklung zu wechseln - einer Normierung, die in den politischpädagogischen Verwendungskontexten des derart erzeugten ,Wissens vom Kind' abgestützt wird." Denn wenn es ein Ziel gibt, auf das der Entwicklungsprozess zuläuft, dann kann (und muss) auch überprüft werden, ob das Kind auf einem guten Weg ist. „Die Notwendigkeit einer kontinuierlichen Beobachtung des Aufwachsens von Kindern wird auf der Grundlage solch eines Entwicklungsparadigmas nachhaltig plausibel gemacht." (Tervooren 2008, S. 43) Zentral für die Popularisierung des Entwicklungsdenkens in der zweiten Hälfte des 19. Jahrhunderts war die Darwinsche Evolutionstheorie. „Ein evolutionistisches Verständnis von ,Entwicklung' betrachtet die Ontogenese als Rekapitulation der Phylogenese, der Gattungsgeschichte, anders gesagt: die kindliche Entwicklung wird als individualgenetischer Nachvollzug der zivilisatorischen Entwicklung aufgefasst." (Honig 1999, S. 61) Vor diesem Hintergrund wurde kindliche Entwicklung zunächst als ein Prozess verstanden, der endogenen Gesetzmäßigkeiten folgt. Turmel (2008) zeichnet in seiner historischen Soziologie der Kindheit 100 Jahre Kindheitsgeschichte (1850 bis 1950), die er als „the apex of the developmental paradigm“ (ebd., S. 2) in den westlichen Industrienationen ansieht, anhand von Quellen aus den USA, England und Frankreich nach. Er bezieht das Entwicklungsparadigma dabei nicht allein auf Kindheit, sondern versteht es als ein übergeordnetes Modell der Geschichte als Fortschrittsgeschichte, die einer vorgezeichneten Abfolge von Phasen und Stufen folgt. Dieses Modell sei ebenso auf Gesellschaften wie auf Individuen anwendbar gewesen (vgl. auch Burman 2007) und habe die Sozialwissenschaften von ihrem Beginn an geprägt. Turmel rekonstruiert wissenschaftliche, gesellschaftliche und technologische Voraussetzungen, die im Laufe des 19. Jahrhunderts eine entwicklungstheoretische Betrachtung von Kindern durchzusetzen erlaubten, und zwar vor allem die Entstehung der Bevölkerungswissenschaften mit ihren Datenerhebungen, Reihenuntersuchungen und Bevölkerungsstatistiken, die auch den Beginn einer empirischen Soziologie markiert. Diese Datensammlungen führten zu Kategorisierungen und Klassifikationen und ermöglichten den methodischen Vergleich von Kindern und damit die Entstehung des modernen Kindheitsbildes.

Zwar liegen die Anfänge systematischer Beobachtungen von Kindern historisch schon früher, am Ende des 18. Jahrhunderts, wie Tervooren (2008) rekonstruiert. Jedoch handelte es sich hier meist um Einzelfallbeobachtungen, etwa wie die von Vätern an ihren eigenen Kindern, die 
an einem von Johann Heinrich Campe 1785 in seiner pädagogischen Enzyklopädie ausgelobten Wettbewerb teilnahmen (vgl. Schmidt 2001); diese Beobachtungen kann man aus heutiger Sicht durchaus als empirische ansehen, aus ihnen wurde allgemeine Erkenntnisse über kindliche Bildungsprozesse abgeleitet. (Und diese Methode erfreute sich auch ein gutes Jahrhundert später bei den ersten deutschsprachigen Entwicklungspsychologen wie Preyer, Stern und Bühler großer Beliebtheit.) Die Grundlagen für sozialstatistisch abgesicherte Entwicklungsdaten lieferten jedoch erst großangelegte Reihenuntersuchungen.

Das Konzept der „Normalität“ hat laut Turmel $(1997,2008)$ für die Betrachtung der Kindheit vor dem Ende des 19. Jahrhunderts noch keine maßgebliche Rolle gespielt. Im Zuge der historischen Etablierung der Kinderkörperstatistik wurden Verfahren zur repräsentativen Erhebung von Kinderkörpernormen im Sinne von Durchschnittswerten und Regelmäßigkeiten für ein bestimmtes Lebensalter überhaupt erst seit der zweiten Hälfte des 19. Jahrhunderts in relevantem Umfang angewandt. Diese Entwicklung führte zur Konfigurierung des „normalen“ Kindes und steht in Zusammenhang mit der - weitgehend sozialpolitisch motivierten Etablierung und Professionalisierung von Kindermedizin, öffentlicher Hygiene und Entwicklungspsychologie. Die moralische Bewertung der Ergebnisse der Reihenuntersuchungen bei Kindern, also deren Übersetzung in Normen im Sinne von sittlichen Ge- und Verboten der Behandlung von Kindern, vollzog sich im Rahmen politischer Auseinandersetzungen um Kinderschutz, Sozialfürsorge und ein Verbot der Kinderarbeit, alles Maßnahmen, die auf eine Senkung der durch Industrialisierung, Verstädterung und mangelhafte Hygiene bedingten Kindersterblichkeit zielten (vgl. auch Klaus 1993). Im Zuge dieser Debatten wurde ein Konzept von kindlicher Entwicklung als vor allem endogen determinierten Prozess im Grunde bereits in Frage gestellt: Denn wenn unterschiedliche Bedingungen des Aufwachsens so massiv auf kindliche Entwicklungsverläufe einwirkten, wie die Ergebnisse der Reihenuntersuchungen es nahelegten, dann war auch die Annahme eines in weiten Teilen vorgezeichneten kindlichen Entwicklungsprozesses hinfällig.

Turmel (2008) zeigt, orientiert an der soziologischen Wissenschaftsforschung und insbesondere der actor-network theory, wie die „Tatsachen“ durchschnittlicher kindlicher Entwicklung der Reflexion und Verwertung überhaupt nur vermittels Repräsentationstechniken wie z.B. verdichteten Visualisierungen in Graphiken, Tabellen und Somatogrammen zugänglich gemacht wurden, die Wirklichkeit in je spezifischer Weise (re)konstruieren. Solchen technologischen Artefakten schreibt die actor-network theory symmetrisch zu den menschlichen Akteuren ebenfalls einen Akteursstatus zu, da sie Praktiken strukturieren, vermitteln und übersetzen. Die historisch ab der Wende zum 20. Jahrhundert als plausibel erachtete normative Bewertung und Skandalisierung von Mortalität und Fehlentwicklungen bei Kindern basierte auf sich verfeinernden wissenschaftlichen Verfahren der statistischen Erhebung und Darstellung, die erst durch die Art, wie sie „Fakten“ repräsentieren, spezifische Argumentationsressourcen für politische Debatten bereitstellten (vgl. auch Lischeid 2001). 
Turmel (2008) erinnert dabei auch an weitere gesellschaftlichen Bedingungen für Reihenuntersuchungen. Die Schulpflicht, man könnte sagen: die massenhafte Versammlung von Kindern in Institutionen, ermöglichte deren pragmatische Durchführung. Wobei Burman (1994, S. 9ff.) darauf hinweist, dass die Anfänge der institutionellen Erziehung von Kindern im Kindergarten zunächst einmal die Möglichkeit bot, mehrere kleine Kinder gleichzeitig zu beobachten, zu vergleichen und auch auf dieser Grundlage Altersnormen für die frühe Kindheit zu schaffen. Im Zuge der Child study Bewegung, die sich zuerst in den USA und dann auch in Europa an der Wende zum 20. Jahrhundert verbreitete, wurde die Beobachtung von Kindern unter mehrfachen Zielsetzungen popularisiert: der Ausarbeitung einer wissenschaftlichen Erziehungslehre, der Entwicklung der Ortho- bzw. Behindertenpädagogik, der Begründung der Schulhygiene und der Modernisierung der Säuglingsfürsorge (vgl. Depaepe 1993, S. 51f.). Diese Bewegung verknüpfte Kindheitsforschung eng mit Erzieher- und Lehrerbildung sowie Sozialpolitik. In den politischen Auseinandersetzungen ging es dabei weniger um die Feststellung von Entwicklungsnormen für sich genommen, als vielmehr um deren Funktionalisierung für die Etablierung von pädagogisch-praktischen Handlungsnormen, die die Entwicklungschancen von Kindern wahren helfen sollten.

Nach dem ersten Weltkrieg verloren Ansätze der experimentellen Pädagogik, der "child study“Bewegung und der Pädologie, die sich von einer vielseitigen empirischen Beobachtung und experimentellen Erforschung der Kinder unmittelbar eine Optimierung der Erziehungs- und Unterrichtspraxis durch so etwas wie „Pädotechnik“ versprachen, international an Bedeutung: Die Hoffnungen auf einen Ansatz, der praktisch-pädagogische und wissenschaftliche Interessen integrierte, erwiesen sich als unrealistisch, und die empirischen Forschungsergebnisse nahmen sich eher bescheiden aus (vgl. Depaepe 1993). In der Folgezeit profilierte sich die Entwicklungspsychologie als (Leit)Disziplin der Kinderwissenschaften, indem sie sich auf die wissenschaftliche Erforschung der kindlichen Entwicklung konzentrierte und deren empirische Methoden rationalisierte. Die entstehende experimentelle und die Testpsychologie paradigmatisch: die Entwicklung von Intelligenztests, um geistig behinderte Kinder identifizieren und institutionell segregieren zu können - arbeitete daran, neben der Erhebung von körperstatistischen Daten, wie sie bei den Reihenuntersuchungen erfasst wurden, weitere Messgegenstände wie etwa Begabungen und Kompetenzen zu konzipieren und dazu Daten zu sammeln.

Vermutlich wurde durch die sukzessive Etablierung aller drei Formen der Erforschung der Entwicklung von Kindern - bevölkerungswissenschaftliche Reihenuntersuchungen, ,klinische' Beobachtungen durch pädagogisches Personal in den entsprechenden Einrichtungen und die Methoden der experimentellen Entwicklungspsychologie - die Vorstellung von Kindheit als Entwicklungskindheit im Verlaufe eines Jahrhunderts auf das Engste mit der Idee einer „normalen“ Kindheit verknüpft. Denn alle drei Formen, insofern sie größere Gruppen von Kindern fokussieren, ermöglichen zentrale Operationen wie unterscheiden, klassifizieren und 
vergleichen, wodurch die Betrachtung des einzelnen Kindes an einer (wenn auch je unterschiedlich dimensionierten) Masse von Kindern relationiert wird.

Die Konstruktion der „normalen“ Entwicklung impliziert dabei stets eine Abgrenzung zur Anormalität (Foucault 2003). Armstrong (1983) zeichnet z.B. für die Entwicklung der Pädiatrie als einer medizinischen Subdisziplin nach, wie diese ausgehend von der Behandlung von Kinderkrankheiten mehr und mehr Zuständigkeiten für die Entwicklung von Kindern insgesamt beanspruchte und dabei eine lange Reihe von Abweichungen wie nervöse, verhaltensgestörte, hypersensible u.a. Kinder mehr produzierte. Die Professionalisierung und Szientifizierung der Kinderwissenschaften insgesamt, so auch Cravens (1985), bildete mit Blick auf die ,anormalen' Kinder die Basis für neue Formen einer interventionistischen Sozial- und Gesundheitspolitik. In diesem Zusammenhang gilt es das oben mit Bezug auf Honig angedeutete unbemerkte Umschlagen der Deskription in Präskription von Entwicklung noch einmal genauer zu betrachten. Leuchtet das Argument für die ,vor-empirischen' Betrachtungen Rousseaus unmittelbar ein, so ist für das Zeitalter von Probabilismus und empirischem Rationalismus zu konstatieren, dass „Normalität“ und „Normativität“ konzeptuell auseinander treten, und zwar gerade durch die wissenschaftlich-technologischen Entwicklungen, die aus modernen Gesellschaften systematisch verdatete Gesellschaften gemacht haben (vgl. Gerhard, Link und Schulte-Holtey 2001). Der Normalismustheoretiker Link (2008) unterscheidet „normalistische Normen“, die statistische Durchschnittswerte und die Definition von „normal ranges“ umfassen, und normative Normen, deren Erfüllung „punktförmig“ erwartet werde. Entsprechend differenziert auch Turmel (2008, S. 183ff.) „soziale Formen der Normalität“: Normalität als Durchschnitt, als Gesundheit und als das Akzeptable oder sogar als Ideal. Durch die historische Differenzierung dieser Dimensionen komme es zu einem „neuen Typ von Interferenzen zwischen Normativität und Normalität", so Gerhard, Link und Schulte-Holtey (2001, S. 7). So lässt sich aus der statistischen Beschreibung kindlicher Entwicklungsnormen nicht unmittelbar die „gute“ Entwicklung ableiten; auf der Basis der Definition von Schwellennormen zur Anormalität werden gleichwohl Normen der Intervention in Entwicklungsprozesse abgeleitet, die nicht einfach ,nur' normativ erscheinen, sondern wissenschaftlich hergeleitet. Auf diese Weise ließen sich im Zuge der Etablierung der Kinderwissenschaften wirkungsvolle Kindheitspolitiken platzieren. Die Veröffentlichung und Verbreitung der Durchschnittswerte für kindliche Entwicklung in Tabellen, Graphiken und Somatogrammen diente seit dem Ende des 19. Jahrhunderts nicht nur erfolgreich dem moralisch begründeten Ziel einer verbesserten Wahrung der Entwicklungschancen von Kindern, sondern trug auch in dem Sinne zur Normierung bei, dass Kinder sich zunehmend an ihnen messen lassen mussten.

Die zitierten historisch-soziologischen und historisch-rekonstruktiven Arbeiten belegen international eine wissenschaftliche Besetzung des Entwicklungsthemas durch Psychologie und Pädiatrie als zentralen Kinderwissenschaften in der Moderne. Vor diesem Hintergrund ist plausibel, dass die gesellschaftliche Bedeutung, die dem Entwicklungsparadigma für die Entstehung der modernen Kindheit zugeschrieben wird, auch in systematischen Arbeiten zur 
Kindheitstheorie durchgängig hohe Aufmerksamkeit erfährt (ex. Honig 1999, S. 59ff.). Auf der Basis dieser historischen und theoretischen Rekonstruktionen der Bedeutungen des Entwicklungsparadigmas für die Genese moderner Kindheit wird im vorliegenden Beitrag dafür plädiert, dass die sozialwissenschaftliche Kindheitsforschung „Entwicklung“ im folgenden v.a. auch mit Fokus auf aktuelle gesellschaftliche Methoden und Verfahren der Beobachtung kindlicher Entwicklung empirisch untersuchen sollte.

\section{Die Kindheitssoziologie und die kindliche Entwicklung}

In modernen Gesellschaften grundieren Vorstellungen von „altersgerechter“ (Hungerland 2003) oder „altersgemäßer“ (Kelle 2007a) Entwicklung maßgeblich die Regulierung des Lebenslaufs und des Aufwachsens von Kindern in Institutionen. Der synonyme Gebrauch von „altersgemäßer“ und „normaler“ Entwicklung macht dabei deutlich, dass sich die normalistischen Normen an denen für ein je bestimmtes Alter durchschnittlich erwartbaren Messgrößen und Kompetenzniveaus orientieren. Diese Normen haben nicht nur dazu geführt, dass Kinder sich je individuell an innen messen lassen müssen und am Individuum orientierte Maßnahmen der Intervention entwickelt wurden, sondern sie haben auch die Formen der Institutionalisierung von Kindheit und Lebenslauf als solche geprägt (vgl. Zeiher in diesem Band). Um z.B. als Kindergarten- oder Schulkind bestehen zu können, müssen Kinder nicht nur, als formales Kriterium, ein bestimmtes Alter erreicht haben, sondern auch Voraussetzungen erfüllen, die in einer altersgemäßen Soziabilität, psychischen Autonomie und Stabilität, Lernwilligkeit und fähigkeit sowie bestimmten Fertigkeiten der Körperbeherrschung bestehen.

Zeiher (in diesem Band) fasst unter der Perspektive der Institutionalisierung und Chronologisierung von Kindheit zusammen: „Das komplex altersgradierte Kindheitsregime wird durch psychologische Konzeptualisierungen der schrittweisen Entwicklung unterstrichen und gerechtfertigt." Zeiher fokussiert damit die Strukturierung von modernen Betreuungs- und Bildungsinstitutionen nach Altersklassen; ein maßgebliches Merkmal dieses Regimes ist es, dass Selektionsentscheidungen im Bildungssystem - wie z.B. die Überweisung an eine Sonderoder Förderschule - mit der Differenz zwischen Alterszugehörigkeit und Entwicklungsstatus, die in entwicklungsdiagnostischen Maßnahmen und Verfahren erhoben wird, begründet wird.

Die gesellschaftliche Verknüpfung des normalistischen Entwicklungsparadigmas mit der Kindheit erfolgt demnach, systematisch betrachtet, in mindestens drei Begründungszusammenhängen: im Kontext der Interventionen in individuelle Entwicklungsverläufe im Sinne von Förderung oder Therapie, im Kontext der altersgradierten Regulierung der Betreuungs- und Bildungseinrichtungen zum Zweck der effektiven Synchronisierung der Lernenden und im Kontext der Begründungspflicht für Selektionsentscheidungen, mit denen Abweichungen von erwartbaren, ,normalen' Entwicklungsverläufen institutionell bearbeitet werden.

Stellt man diese systematischen Überlegungen in Rechnung, so ist festzuhalten, dass die auf die Gegenwart bezogene empirische Kindheitssoziologie sich dem Gegenstand der kindlichen Entwicklung bisher noch nicht angemessen gewidmet hat. Am Ausgangspunkt der neueren 
Kindheitsforschung stand die Kritik an einer entwicklungspsychologisch grundierten

Sozialisationsforschung: Kritisiert wurde deren implizite Orientierung hin auf "reife“, erwachsene Entwicklung als eine adultistische Perspektive (vgl. auch Honig in diesem Band). Mag diese Kritik am Ausgangspunkt der new social studies of childhood aus heutiger Sicht auch überspitzt gewesen sein, diente sie doch der Profilierung der eigenen Fokussierung auf die relative Eigenständigkeit und vor allem je gegenwärtige soziale Bedeutsamkeit kindlicher Aktivitäten. Die Dichotomisierung von Kindern und Erwachsenen mit Bezug auf Kompetenzen lehnten die Kindheitssoziologen ab und setzten ihr die Prämisse entgegen, dass Kinder von Beginn an soziale Akteure sind. Sie begriffen Kindheit nicht mehr als Phase der Hervorbringung zukünftiger Gesellschaftsmitglieder, sondern als je gegenwärtig wirksame soziale Strukturkategorie und diskursives Konstrukt (Zeiher 1996; Alanen 2000).

Dieser Wechsel von einem diachronen Blick, wie inn Sozialisationstheoretiker und Entwicklungspsychologen einnehmen, hin zu einer synchronen soziologischen Perspektive auf Kinder als gesellschaftliche Gruppe, mag nun seinerseits die Aufmerksamkeit für eine soziologische Re-Theoretisierung der kindlichen Entwicklung verzögert haben. Die Akzente der mikrosoziologischen Kinderforschung lagen zunächst in den Sozialsystemen, Interaktionsordnungen und kommunikativen Praktiken von Kindern, beginnend etwa beim Kindergartenalter (ex. Corsaro 2005), und dem Konzept der „interpretive reproduction“, über die sich Kinder in ihren peer groups selbst sozialisieren. Obwohl es sich hierbei häufig um Ethnographien von peer cultures in Institutionen handelte, wurde der Umstand, dass deren Altersgradierung eine spezifische Form institutioneller peer cultures überhaupt erst schafft, mehr als selbstevident genommen denn zum analytischen Gegenstand gemacht (vgl. jedoch Kelle 1997 und 2001). - Ein Schwerpunkt der strukturorientierten Kindheitsforschung liegt in dem wachsenden Problem der Kinderarmut und der Entwicklung einer adäquaten Kindersozialberichterstattung (Joos 2001, Leu 2002, Betz 2008). Im Kontext der Sozial- und Gesundheitsberichterstattung wird seit einigen Jahren durchgängig über die Probleme der wachsenden gesundheitlichen Beeinträchtigungen und Entwicklungsverzögerungen bei sozial benachteiligten Kinder berichtet (ex. Robert Koch-Institut 2007). Diese Befunde basieren auf multidisziplinären Wissensbeständen zu altersgemäß erwartbaren Entwicklungsstatus - sie hinterfragen das gesellschaftliche Konstrukt der „altersgemäßen Entwicklung“ nicht, sondern setzen es voraus und bedienen es.

Zwar ist in kindheitssoziologischen Arbeiten vielfach darauf hingewiesen worden, dass die binäre Konstruktion „Kinder-Erwachsene“ Kindheit naturalisiert und universalisiert (ex. James et al. 1998). Allerdings führt die Grundsatzkritik und Perspektiven der Re- und Dekonstruktion in Hinblick auf die ,Natürlichkeit' der kindlichen Entwicklung (Burman 1994; Mayall 1996; Woodhead 1999) erst allmählich dazu, weitere empirische Perspektiven zu informieren. Gleichzeitig wird die Materialität des Kinderkörpers kindheitssoziologisch ,wiederentdeckt' (James 2000; Christensen 2003; Hengst und Kelle 2003). Prout (2000) macht für die zukünftige Forschung deutlich, dass die Kindheitssoziologie dem biologischen Reduktionismus und 
Substantialismus anderer wissenschaftlicher Körperkonzeptionen nicht einfach einen kulturellen Reduktionismus an die Seite stellen, sondern die materielle Spezifität von Kinderkörpern sowie die unterschiedlichen - diskursiven, technologischen u.a. - Ressourcen, die zu ihrer Konstruktion beitragen, ernstnehmen sollte. Kinderkörper und kindliche Entwicklung nicht als „natürliche“ Entitäten, sondern als Netzwerke von heterogenen Materialien und Ressourcen zu verstehen ist bislang v.a. in historischer Perspektive (Turmel 2008) und für hochtechnisierte Kontexte wie Intensivstationen plausibel gemacht worden (Place 2000; vgl. jedoch Bollig 2008 für eine Ausweitung dieser Perspektive auf den Bereich der präventiven Entwicklungsdiagnostik).

Darüber hinaus ist die kindheitssoziologische Kritik an entwicklungspsychologischen, sozialisationstheoretischen und pädiatrischen Perspektiven inzwischen zu differenzieren. Zum einen hat Honig (1999, S. 63) bereits darauf hingewiesen, dass kontextuelle (z.B. Bronfenbrenner) und strukturgenetische (z.B. Edelstein/Nunner-Winkler) Ansätze von einer kindheitssoziologischen Grundsatzkritik insofern nicht getroffen werden, als sie „sich der versteckten teleologischen und ethnozentrischen Implikationen des Entwicklungskonzepts besonders bewusst“ seien. „Die neuere entwicklungsgenetische Forschung nimmt eine differentielle Perspektive ein. Sie ist an interindividuellen Unterschieden (,Individualität') interessiert und betont die Vielfalt der Entwicklungsbedingungen und -perspektiven gegenüber durchschnittlichen Entwicklungsverläufen“ (ebd., S. 64). Zudem liegen zunehmend Kritiken am universalistischen Kindheitsbild der (früheren) Entwicklungspsychologie aus dem Kontext der Entwicklungspsychologie selbst vor (vgl. Burman 2007; Kontopodis 2007; Woodhead 2009), die ebenfalls deutlich machen, dass sich diese Disziplin der Probleme der Komplexitäten heutiger Kindheiten und pluraler Entwicklungsbedingungen und -verläufe annimmt. „In short, a strong research agenda on change and transitions in children's growth, learning and well-being (including children's perspectives on these themes) is essential for Childhood Studies, even if we choose no longer to describe this agenda as about ,child development'" (Woodhead 2009, S. ...). Die kindheitssoziologische Kritik am „developmentalism“ würde vor diesem Hintergrund dann in der Gefahr stehen, sich für die interdisziplinäre Diskussion zu disqualifizieren, wenn sie in veralteten Klischees von anderen kinderwissenschaftlichen Disziplinen verharrte. Zum anderen verweist Turmel (2008) auf historische Arbeitsteilungen zwischen Disziplinen, an denen die Soziologie durchaus selbst partizipiert hat. In klassischen Sozialisationskonzeptionen bei Durkheim, Parsons, aber auch noch bei Bourdieu referierten die Autoren unkritisch auf formale entwicklungspsychologische Konzepte und hätten in der Soziologie selbst ein Kindheitsverständnis befördert, das im Kind nichts anderes als ein Wesen, das zur Erwachsenheit heranreift, sehen könne (Turmel 2008, S. 18f.; vgl. auch Zeiher 1996); Turmel geht mit Bezug auf die klassischen Sozialisationstheorien ähnlich wie Zeiher soweit, Kinder und Kindheit als ,sociology's unthinkable object' zu bezeichnen. Fuhs (2003) beklagt für die komplementäre Aufteilung von disziplinären Zuständigkeiten zudem, dass die Kindheitsforschung sich tendenziell mit den kompetenten und die Kindermedizin mit den 
kranken und entwicklungsgestörten Kindern befasse. Letztlich weist er damit auf die implizite Normativität der kindheitssoziologischen Forschung hin, der analytisch jedoch nicht beizukommen ist, wenn nachholend auch die bisher nicht gebührend berücksichtigten „unruhigen“, „behinderten“, „entwicklungsverzögerten“ oder „dicken“ Kinder in den Blick geraten - abgesehen davon, dass die Kritik insofern nicht ganz zutreffend ist, weil sich Soziologen und Kindheitsforscherinnen auch schon früh mit der sozialen Konstruktion von abweichender Entwicklung beschäftigt haben (vgl. ex. Conrad 1976 zu ADHS; Bühler-Niederberger 1991 zu Legasthenie). Vielmehr gilt es, die Analysen auf die Normativität der unterscheidenden Entwicklungslogik und der (diagnostischen) Diskurse und Praktiken zu richten, die Differenzen zwischen Kindern nicht einfach ,feststellen', sondern ko-produzieren.

Zum Stand der kindheitssoziologischen Forschung zu Kinderkörpern und Entwicklung ist demnach festzuhalten, dass bislang eine in empirischer Forschung gründende Soziologisierung von „Entwicklung“ fehlt, die der Diskontinuität der körperlichen Existenz, der Variabilität der körperlichen und kompetenzbezogenen Wandlungsprozesse und den diskursiven, praktischen und institutionellen Netzwerken, in denen Kinderkörper sich formieren, im Sinne einer „sociology of embodiment" (Featherstone und Hepworth 1998; James 2000) theoretisch Rechnung tragen würde. An diesem Befund ändern auch neuerdings aufkommende Ansätze, die Kinder - um den Paradigmenstreit zu überwinden - dialektisch sowohl als „beings“ wie auch als „becomings“ (ex. Uprichard 2008) begreifen, noch nicht wirklich etwas, da sie eher in konzeptioneller Perspektive auf den Aspekt der Temporalisierung von Kindheit eingehen, statt eine empirisch fundierte Soziologie der kindlichen (Körper-)Entwicklung zu verfolgen.

\section{Die Institutionalisierung von entwicklungsdiagnostischen Verfahren in der frühen Kindheit und die Early Social Childhood Studies}

In den letzten Jahren entstanden vermehrt interaktions-, performanz- und habitustheoretische Arbeiten in der Kindheitsforschung, die sich dem Thema Kinder und Körper widmen (ex. Prout 2000; Hengst und Kelle 2003; Tervooren 2006) und Kinder (und Jugendliche) im Schulalter in den Vordergrund stellen. Den Social Studies of Childhood wird vorgeworfen, den Schwerpunkt ihres Interesses v.a. bei den 6- bis 12-Jährigen zu setzen. Die frühe Kindheit dagegen wurde bisher selten zum Gegenstand rekonstruktiver Studien und verblieb weitgehend im Zuständigkeitsbereich von entwicklungspsychologischen und frühpädagogischen Ansätzen. Dabei stellt die frühe Kindheit gegenwärtig ein Feld dar, in dem studiert werden kann, wie die oben unterschiedenen systematischen Begründungszusammenhänge, in denen das normalistische Entwicklungsparadigma eine maßgebliche Rolle für die Konstitution von Kindheit spielt -Interventionen in individuelle Entwicklungsverläufe, altersgradierte Regulierung der Betreuungs- und Bildungseinrichtungen und Begründungen für Selektionsentscheidungen - in neuen Institutionalisierungsformen zusammenlaufen und zunehmend enger verkoppelt werden. Immer mehr Kinder werden in verschiedene, z.T. staatlich regulierte Formen der Begutachtung 
ihrer Entwicklung einbezogen. An der Feststellung von Entwicklungsstatus der Kinder sind heute in der Regel eine Reihe von Professionen beteiligt, insbesondere Kindermediziner, Entwicklungspsychologen, Sprachwissenschaftler, Logopäden und andere sowie verschiedene professionelle Pädagogen, die untereinander zunehmend enger vernetzt werden; neben den Professionellen spielen selbstverständlich auch die Eltern, in Interaktion mit den Professionellen, eine Rolle in den Prozessen der Beobachtung der Kinder. Der Status der kindlichen Entwicklung ist demnach eine ,Tatsache', die im Rahmen (fachlicher) Wissensordnungen und -kulturen (vgl. Knorr-Cetina 2002; Law und Mol 2002) sowie deren praktischer Vernetzung konfiguriert wird. Die Entwicklung von Kindern erfährt demnach in bildungs- und gesundheitspolitischen Diskursen um die Prävention von Entwicklungsstörungen, um Frühförderung und Bildungspläne für das (Vor-)Schulalter verstärkt öffentliche Aufmerksamkeit. So haben z.B. alle 16 deutschen Bundesländer in den vergangenen Jahren nach einem von der Kultusministerkonferenz 2004 veröffentlichten Rahmenplan Bildungspläne für den Elementar- und z.T. Primarbereich vorgelegt. Für den Bereich der frühpädagogischen Praxis gibt es inzwischen differenzierte Beobachtungs- und Dokumentationsverfahren, mit denen Erzieherinnen die in den Bildungsplänen geforderte systematische Dokumentation der Entwicklungs- und Bildungsprozesse der Kinder professionell umsetzen können (vgl. Leu 2008). Im öffentlichen Diskurs ist darüber hinaus vielfach von „Frühwarnsystemen“ in Hinblick auf Gefährdungen der kindlichen Entwicklung die Rede, es geht dabei um eine Bündelung von Maßnahmen und die Institutionalisierung einer engmaschigen Beobachtung von Kindern von Geburt an. Gemeinsam ist den durchaus unterschiedlichen Positionen in diesen Debatten der Wille und das Bekenntnis zur fördernden Intervention in kindliche Entwicklungsprozesse, und zwar so früh wie möglich. Denn in den letzten 20 Jahren haben Entwicklungsbeeinträchtigungen im Kindesalter laut den einschlägigen Statistiken international stark zugenommen (ex. für Deutschland RKI 2007). Es entstanden neue Störungsbilder, entwicklungsdiagnostische Screenings (international ex. Frankenburg et al. 1992; Bayley 2005; American Academy of Pediatrics et al. 2006) und differenziertere Diagnoseinstrumente, z. B. für die Bereiche Verhaltens-, emotionale und psychische Auffälligkeiten, Sprach-, Sprech- und Stimmstörungen, grob- und feinmotorische Defizite sowie Aufmerksamkeitsprobleme (vgl. ex. Macha und Petermann 2006), mit besonderem Fokus auf der frühkindlichen Entwicklung. Die internationale Standardisierung der Klassifizierung von (funktionellen) Entwicklungsstörungen ist ebenso vorangeschritten (vgl. ICD10, WHO 1993) wie die Institutionalisierung von internationalen epidemiologischen Untersuchungen (ex. WHO 2006).

In vielen Ländern werden inzwischen im Rahmen von kindermedizinischen Vorsorgeuntersuchungen entwicklungsdiagnostische Beobachtungen und Verfahren routinemäßig und institutionalisiert durchgeführt. So gibt es z.B. in Deutschland ein durch das Sozialgesetzbuch $\vee$ abgesichertes Programm mit 10 Vorsorgeuntersuchungen (U1 bis U9) von Geburt bis zum Alter von 5 Jahren; in der Schweiz werden insgesamt 12 Vorsorgeuntersuchungen für Kinder (acht davon im Vorschulalter) durchgeführt; in England gibt 
es ein Programm mit dem Namen "health for all children“, das 15 Untersuchungen bis zum fünften Lebensjahr umfasst; in Österreich werden insgesamt elf Untersuchungen durchgeführt, die direkt nach der Geburt beginnen und mit dem 62. Lebensmonat enden. Solche Verfahren stellen Formen der staatlichen Institutionalisierung von Früherkennung und Entwicklungsbeobachtung dar, die konzeptionell auf die Einbeziehung aller Kinder ausgerichtet sind.

Grundsätzlich lässt sich für solche Maßnahmen sagen, dass sie das Beobachten, Messen und Beurteilen der Entwicklung des je einzelnen Kindes und der Kinder in der Masse in historisch neuer Weise koordinieren, synchronisieren und praktisch aneinander binden; sie überbieten darin deutlich die von Turmel für das 19. und frühe 20. Jahrhundert rekonstruierten Reihenuntersuchungen. Das Ziel solcher Maßnahmen ist in individualmedizinischer und pädagogischer Hinsicht die Früherkennung von Störungen und Feststellung von auf das einzelne Kind bezogenem Frühförderbedarf. In epidemiologischer Hinsicht ist eine Erfassung möglichst aller Kinder der je untersuchten Altersgruppe das Ziel, und zwar zum einen in Hinblick auf die Gesundheitsberichterstattung und zum anderen auf die Feststellung von Förderbedarf bezogen auf die ganze untersuchte Population, um entsprechende staatliche Förderprogramme auflegen zu können. Während für die Hervorbringung deskriptiver Entwicklungsnormen, an denen Kinder gemessen werden können, repräsentative Stichproben als ausreichend gelten, haben Totalerfassungen für die Epidemiologie noch einen anderen Stellenwert: Sie ermöglichen es, Daten zum gesundheitlichen Zustand einer Altersgruppe unselektiert zu gewinnen. Diese Daten gelten als zuverlässiger, da bei ihrer Erhebung auf die immer schon in die Konstruktion von „Repräsentativität“ einfließenden Selektionskriterien verzichtet wird. Das Ziel einer (nahezu) 100\%igen Erfassung aller Kinder wird in Deutschland bisher jedoch nicht von den U1 bis U9, sondern von den ärztlichen Schuleingangsuntersuchungen erreicht, die schulgesetzlich geregelt sind und ca. 3-10 Monate vor der Einschulung erfolgen. Diese bilden die statistische Grundlage für die seit den 1990er Jahren etablierten Kindergesundheitsberichte der Bundesländer (vgl. ex. Bayern 2006; Berlin 2006; Hessen 2006).

Diese Berichte offenbaren in ihren Ergebnissen die große Heterogenität der Entwicklungsstände und Lernvoraussetzungen bei den Einschülern. Neben den analogen klinischen Beobachtungen zur Entwicklungsheterogenität durch Erzieherinnen und Lehrkräfte fordern diese statistischen Ergebnisse die Tradition der Altersgradierung der institutionellen Erziehung und Bildung heraus: Denn wenn nicht mehr 5\%, sondern 25\% der Kinder die Erwartungen an ihre altersgemäße Entwicklung in bestimmten Kompetenzbereichen wie z.B. der Sprachentwicklung unterbieten, dann haben Institutionen, die mit der relativen Altershomogenität meinten eine relative Entwicklungshomogenität unterstellen zu können, ein Problem.

Die Normalisierung der Kindheit und die Ambivalenz von „Heterogenität und Standardisierung der kindlichen Entwicklung“ (Kelle und Tervooren 2008) kann man vor diesem Hintergrund als einen aktuellen Forschungsschwerpunkt der early childhood studies konzipieren. In den Institutionen des Elementar- und Primarbereichs - aber auch darüber hinaus - hat sich der 
Begriff Heterogenität zu einem zentralen der pädagogischen Rhetorik entwickelt: Die Unterschiedlichkeit von Kindern wird entlang der Differenzachsen Geschlecht, ethnische und religiöse Zugehörigkeit, Herkunftsmilieu u.a.m. reflektiert und auf unterschiedliche Dimensionen (körperliche, kognitive, sprachliche, psychische, soziale) der Entwicklungs- und Bildungsprozesse der Kinder projiziert. Zum einen deutet die Heterogenitätsrhetorik auf eine Entgrenzung des Normalitätsparadigmas hin: Das Konzept der „Inklusion“ in sonder- und migrationspädagogischen Ansätzen z.B. verweist auf Bemühungen um eine pädagogische Normalisierung aller Kinder und eine nichtselektive Logik. Zum anderen hat kein institutioneller Bereich der Kindheit in der jüngsten Zeit international einen derartigen Ausbau und Institutionalisierungsschub erfahren wie die Maßnahmen zur Früherkennung und Prävention von Entwicklungs- und Lernstörungen, die notwendig nach wie vor standardisierte Vorstellungen von altersgemäß entwickelten Lernvoraussetzungen zugrunde legen (müssen).

Die Ausprägungen dieses Institutionalisierungsschubs sind in Deutschland z.B. an der aktuellen, länderübergreifenden Diskussion um die gesetzliche Verpflichtung der Eltern zu Kindervorsorgeuntersuchungen (U1 bis U9) abzulesen. Einige Bundesländer wie etwa Bayern, Brandenburg, Bremen, Hessen, Niedersachsen, Rheinland-Pfalz, Saarland und SchleswigHolstein haben mit hohem Durchsetzungstempo 2006/2007 Gesetze novelliert, die den U1 bis U9 Kontrollfunktionen im Sinne des wohlfahrtsstaatlichen Wächteramtes über das Kindeswohl zuschreiben, die zuvor nicht in die Zuständigkeit von Kindermedizinern fielen. Im Zuge der Etablierung eines verbindlichen Einladewesens soll dabei an neu eingerichtete ScreeningStellen gemeldet werden, welche Kinder die Vorsorgetermine wahrgenommen haben, diese Daten werden mit den Melderegistern verglichen, um säumige Eltern zu ermitteln. Kommen diese der Aufforderung, ihre Kinder zu den Untersuchungen vorzustellen, nicht nach, wird die Nicht-Teilnahme selbst als Indiz für Gesundheits- und Entwicklungsgefährdungen des Kindes deutbar und zieht Interventionen von Mitarbeitern der Jugend- oder Gesundheitsämter nach sich (vgl. z. B. Hessischer Landtag 2007; vgl. zur engen Kopplung von Vorsorge und Kontrolle zusammenfassend Bollig und Kelle 2009).

Nicht zufällig forcieren staatlich institutionalisierte Entwicklungsbeobachtungen und Reihenuntersuchungen in den Ergebnissen, die sie hervorbringen, tendenziell traditionelle Differenzachsen: Kinder mit Migrationshintergrund und anderen sozialen Benachteiligungen wie Armut seien überproportional von Gesundheits- und Entwicklungsrisiken betroffen (vgl. Kinderund Jugendgesundheitssurvey KIGGS, RKI 2007). Diese Ergebnisse lassen erahnen, dass die pädagogische Heterogenitäts- und Individualisierungsrhetorik es nicht vermag, das gesellschaftliche Spannungsfeld von Heterogenität und Standardisierung, das durch die Formen der institutionellen Beobachtung der Kinder mitkonstituiert wird, aufzulösen.

Ein zweiter Forschungsschwerpunkt betrifft die Ambivalenz von Selbststeuerung und Disziplinierung, die der institutionellen Betreuung von Kindern diskursiv eingeschrieben ist. Auf der einen Seite dominieren mächtige Normen der kindlichen Selbsttätigkeit, Selbstständigkeit und Selbststeuerung das pädagogische Feld: So betonen etwa die von den Bundesländern in 
den vergangenen Jahren herausgegebenen Bildungspläne für den Elementar- und Primarbereich im Anschluss an die neue Säuglingsforschung, dass bereits Säuglinge aktiv Interaktionen mitgestalten und insofern als Akteure in eigenem Recht wahrzunehmen seien, und sie betonen insgesamt das Bild von Kindern als aktiven Ko-Konstrukteuren ihrer Bildungsprozesse (ex. Hessen 2007).

Die Prozesse des Umbaus des Elementar- und Primarbereichs - massiver Ausbau der vorschulischen Betreuung und Bildung mit Verzahnung und Kooperation von Elementar- und Primarbereich (Krippenplätze, Bildungspläne u.a.) - lassen sich auf der einen Seite als ,weiche' Steuerungsversuche interpretieren, bei denen rigide Formen der Altersgradierung von Institutionen eher aufgeweicht werden. Exemplarisch sei hier auch auf die Kritik an den Rückstellungsquoten bei den Einschulungen und das Konzept der flexiblen Schuleingangsstufe verwiesen, bei dem auf eine Schuleingangsselektion verzichtet und in alters- und entwicklungsheterogenen Gruppen gelernt wird. Der Umbau wird von Kindheitsforschern auf der anderen Seite, mit Bezug auf das Konzept der Gouvernementalität von Foucault und die Ebene der agency von Kindern, doch auch als zunehmend subtilere und zunehmend frühere Formen der Sozialdisziplinierung (Hendrick 2003) und Inkorporierung von Kontrolle interpretiert. Warming Nielsen und Kampmann (2007) weisen auf das Paradox hin, dass die pädagogische Norm der Selbststeuerung Kinder mit dem Risiko konfrontiert, den erwarteten Verhaltensstandards nicht zu entsprechen und in der Folge mit dem Risiko, dass die Berechtigung ihrer Zulassung und Zugehörigkeit zu Institutionen in Zweifel gezogen werden kann. Diese Autoren identifizieren neue Typen von Exklusionsmechanismen, die mit dem soziokulturellen Hintergrund und milieugebundenen Habitus von Kindern und Familien auf das Engste verknüpft sind.

Die Akzente dieser beiden Forschungsschwerpunkte, die es weiter auszubauen gilt, lassen sich mit Bezug auf die oben genannten Begründungszusammenhänge des normalistischen Entwicklungsparadigmas und die zuletzt beschriebenen Entwicklungen wie folgt zusammenfassen: In Hinblick auf Interventionen in individuelle Entwicklungsverläufe im Sinne von Förderung und/oder Therapie zeigt sich erstens eine Tendenz der Vorverlagerung von Maßnahmen in die frühe bis sehr frühe Kindheit. In Hinblick auf die altersgradierte Regulierung der Betreuungs- und Bildungseinrichtungen zum Zweck der effektiven Synchronisierung der Lernenden zeigt sich zweitens, dass es - als Reaktion auf die relative Entkoppelung von Alter und Entwicklungsstand - teilweise auch zu einer Entkoppelung der Frage der Synchronisierung der Lernenden von der Frage der Homogenität der Lernvoraussetzungen kommt; in altersgemischten Settings (nicht nur in der Schuleingangsstufe, sondern auch darüber hinaus) vergrößern Pädagogen bewusst die ohnehin anzunehmende Heterogenität der Lerngruppe und gehen mit dem Einsatz von differenzierten Lehr- und Lernmethoden gar nicht mehr von der prinzipiellen Synchronisierbarkeit der Lernenden aus. Dennoch konstituieren viele Maßnahmen der Beobachtung und Begutachtung der kindlichen Entwicklung drittens ein ambivalentes Feld zwischen optimierter Förderung und Selektion: Dass sie nach wie vor auch zur Begründung von 
Selektionsentscheidungen dienen, zeigt sich an kaum gesunkenen Quoten der Zurückstellung von der Einschulung, frühen Sonder- bzw. Förderschulüberweisungen oder z.B. in Hessen bei der Zuweisung von Kindern mit Migrationshintergrund in so genannte Vorlaufkurse elf Monate vor der Einschulung, in denen sie ihre Deutschkenntnisse verbessern sollen. Immer frühere Interventionen in kindliche Entwicklungsprozesse genießen im öffentlichen Diskurs eine hohe Legitimität; die Ambivalenz von einer immer früheren Förderung und einer immer früheren Stigmatisierung von Kindern als förderbedürftigen ist demgegenüber noch nicht ausreichend analytisch beleuchtet worden.

Dass die Maßnahmen der fürsorglichen Entwicklungsbeobachtung und -begutachtung, die sich im Zuge der Geschichte der Wohlfahrtsstaaten entwickelt und ausdifferenziert haben, in „education, education, education" unter staatlicher Aufsicht münden, darauf weist Hendrick (2003) in seiner Analyse der child care policy von New Labour in Großbritannien hin, die er als „Panoptizismus im Dienste des Kommunitarismus“ deutet. Durch die Analyse der Institutionalisierungsformen der Kindheit und der generationalen Ordnung wird die immanente pädagogische Thematisierung von normativen Konzepten wie z.B. Selbstständigkeit, Inklusion oder Individualisierung obsolet; vielmehr gilt es für die Kindheitsforschung, solche Konzepte immer schon in ihren jeweiligen institutionellen Formen und politischen Rahmungen analytisch zu kontextualisieren.

\section{Fazit: Aufgaben einer (empirischen) Soziologie der kindlichen Entwicklung und Entwicklungsbeobachtung}

In strukturtheoretischer Sicht verweist die verdichtete Institutionalisierung der (frühen) Kindheit auf eine Transformation im Selbstverständnis von modernen Wohlfahrtsstaaten hin zu so genannten „Sozialinvestitionsstaaten“ (vgl. Wintersberger u.a. 2007; Olk 2007), die in Kinder als „Humankapital“ frühzeitig investieren, um die eigene Zukunft zu sichern. Eine der konzeptuellen Herausforderungen für die Kindheitsforschung, die sich aus diesen neueren Entwicklungen ergibt, liegt in der Wiederkehr bzw. Betonung des Konzepts von Kindern als zukünftigen Erwachsenen, Bürgern und Erwerbstätigen. Haben die social studies of childhood in den letzten 20 Jahren auch den Perspektivwechsel hin zu Kindern als je gegenwärtigen Akteuren und Mitgliedern der Gesellschaft befördert, so muss sich die Kindheitsforschung aktuell wieder mit der gesellschaftlichen Wirkmächtigkeit des Dispositivs der Kindheit als „Entwicklungskindheit“ auseinander setzen, nach dem sich die Bestimmung der Kinder in der Zukunft erfüllt. Die gesellschaftlichen Bezüge, in denen dieses aus der Geschichte der Kindheit bekannte Dispositiv zu sehen ist, haben sich allerdings grundlegend gewandelt. Die politischen Bemühungen um eine internationale Standardisierung der Bedingungen des Aufwachsens und der vorschulischen wie schulischen Bildung lassen sich als eine Wiederkehr universalistischer Kindheitsbilder mit anderen Mitteln, als sie der klassischen Pädagogik zur Verfügung standen, interpretieren, nämlich den Mitteln institutioneller Regulierung über Staatsgrenzen hinweg. Die Thesen des Neo-Institutionalismus zur „world polity“ besagen, dass sich z.B. in den Bereichen 
Bildung, Recht oder Umweltschutz „westlich geprägte“ kulturelle Ordnungen international durchsetzen (Hasse und Krücken 2005). Gerade auch die im Bereich der Kindergesundheitsund -vorsorgepolitik zu beobachtenden internationalen Standardisierungen verweisen auf ein tendenziell globales Regime der Institutionalisierung der (frühen) Kindheit, das sich durch Orientierung an den in westlichen Gesellschaften entwickelten normalistischen Modellen kindlicher Entwicklung auszeichnet, für die statistische normal ranges konstitutiv sind. Vor dem skizzierten Hintergrund müsste sich die neuere sozialwissenschaftliche Kindheitsforschung dem Gegenstand der „kindlichen Entwicklung“ nicht nur mit Bezug auf den Umbau der Wohlfahrtsstaaten (ex. Kränzl-Nagl, Mierendorff und Olk 2003), sondern auch in wissenssoziologischer und praxisanalytischer Mikroperspektive widmen, um die oben zitierten verdienstvollen historischen Arbeiten um Analysen zu bereichern, die sich auf aktuelle gesellschaftliche Entwicklungen beziehen. In kultur- und praxisanalytischer Perspektive können z.B. kindliche „Kompetenzen“, wie sie als Voraussetzungen für den Kindergartenbesuch charakterisiert wurden, auf Distanz gebracht werden und es kann danach gefragt werden, wie sich die Attribuierung bestimmter Fähigkeiten an das Alter - und in deren Folge die Diagnose eines bestimmten Entwicklungsstandes eines Kindes - in situierten Prozessen kulturell vollzieht. Es gilt eine Forschung weiter zu entwickeln, die unter der Perspektive von "doing difference“ (vgl. Kelle 2005) entwicklungsdiagnostische Klassifikationen und Verfahren als soziale Praktiken der Unterscheidung analysiert, die institutionelle Förderungen wie auch Selektionen und Bildungslaufbahnentscheidungen maßgeblich informieren und legitimieren.

Für diese Forschung ist - insbesondere auch mit Blick auf die Frage nach der (welt)kulturellen Durchsetzung von Institutionen der Vorsorge - allerdings die Unterscheidung von wissensbasierten Artefakten, die institutionelle Ordnungen strukturieren (wie z.B. die erwähnten Entwicklungstabellen und Somatogramme), und einer gelebten, praktisch-kulturellen Ordnung interessant. Denn die Instrumente determinieren Vollzug und Ergebnisse der diagnostischen Verfahren nicht, oder jedenfalls nicht eindeutig oder vollständig. Vor diesem Hintergrund wäre auch Turmels (2008) Wertschätzung von graphischen Visualisierungen für die historische Durchsetzung des Entwicklungsparadigmas noch einmal quellenkritisch zu hinterfragen - er hatte methodisch nicht die Chance, den Gebrauch dieser Instrumente in der Praxis zu beobachten. Im Unterschied zur Beschränkung der Historiker auf vorfindliche Quellen haben die auf die Gegenwart bezogenen erfahrungswissenschaftlichen und diskursanalytischen Forscher allerdings den Vorteil, dass innen ein ausdifferenziertes Arsenal an Forschungsmethoden die Erzeugung und Sammlung reichhaltigen Materials ermöglicht, das die variantenreiche Anwendung von diagnostischen Instrumenten und Verfahren in der Praxis zeigen kann.

Eine solche Perspektive wird derzeit im Frankfurter Projekt „Kinderkörper in der Praxis. Eine Ethnographie der Prozessierung von Entwicklungsnormen in Kindervorsorge- und Schuleingangsuntersuchungen“ umgesetzt. An diesem Projekt lassen sich exemplarisch die folgenden Gegenstandsebenen unterscheiden: 
- Es kann nach der diskursiven, politischen und institutionellen Konfigurierung der staatlich regulierten Maßnahmen der Entwicklungsbeobachtung und -diagnostik bei Kindern gefragt werden (ex. Kelle 2008 für Vorsorge- und Schuleingangsuntersuchungen);

- Es kann die Durchführung von Verfahren der Entwicklungsbeobachtung auf der Mikroebene des Geschehens mit Mitteln ethnographischer Beobachtung und Analyse rekonstruiert werden (ex. Bollig und Ott 2008, ebenfalls Vorsorge- und Schuleingangsuntersuchungen);

- Es kann zwischen der Konstruktionslogik von (diagnostischen) Instrumenten und ihrem Gebrauch in der Praxis unterschieden werden; Dokumenten- und Instrumentenanalysen sowie Analysen des Gebrauchs von Dokumenten und Instrumenten können auf diese Weise systematisch aufeinander bezogen werden (ex. Kelle 2006 für Sprachtests; Kelle 2007b für Somatogramme; Bollig 2008 zu „Praktiken der Instrumentierung“ in methodologischer Perspektive).

- Und es können schließlich die Teilnehmersichtweisen auf die verschiedenen praktischen Ebenen der Entwicklungsbeobachtung und -diagnostik in ethnographischen und Experteninterviews rekonstruiert werden.

Bollig und Ott (2008) haben in ihrer Teilstudie z.B. gezeigt, dass sich Vorsorgeuntersuchungen in ihrer Durchführung durch ein komplexes „praktisches Management der Normalität“ auszeichnen, bei denen an Altersgruppen normierte Modelle zwar die medizinische Wahrnehmung des Kinderkörpers maßgeblich prägen und die praktische Realisierung und Einordnung von Befunden erst ermöglichen. Wenn es jedoch um die explizite Zuschreibung von Abweichungen geht, zeigen sich vielfach Strategien der Verflüssigung von Normalitätsgrenzen, indem die Unterscheidungen zwischen normaler und gestörter Entwicklung unter Verweis auf mögliche individuelle Variationen in der Schwebe gehalten werden. Um Gefährdungspotentiale dabei nicht aus dem Blick zu verlieren, wird meist eine intensivierte Beobachtung und Kontrollfrequenz als erforderlich erachtet, womit die „Arbeit an der Grenze“ zwischen normaler und gestörter Entwicklung im Rahmen der Vorsorgeuntersuchungen auf Dauer gestellt und ein diffuses Risikobewusstsein verstetigt wird.

Besonders spannend in Bezug auf die genannten Gegenstandsebenen ist ihre methodologische Verknüpfung in komplexen Forschungsdesigns. Institutionelle Beobachtungen und diagnostische Untersuchungen der „Entwicklung“ von Kindern tendieren dazu, ihr Programm "from normalization to optimization" (Nadesan 2008; vgl. auch Kelle und Ott 2009) umzustellen. Weitere praxisanalytische Studien in diesem Bereich können von gouvernementalitätstheoretischen Arbeiten wie der Nadesans profitieren, wenn es darum gehen soll, die Ebene der mikropolitischen Durchführung in situ mit den Ebenen der diskursiven und politischen Konfigurierung zu verknüpfen (vgl. auch Bühler-Niederberger 2005). In einer anderen, aber ähnlichen Argumentationslinie betont Smiths (2002) Ansatz einer „institutional ethnography“ die Verbindungen zwischen den Situationen und Lokalitäten des alltäglichen Lebens, professionellen Praktiken und der Politik. 
Und schließlich könnte solch eine Forschungsperspektive über den Bereich von entwicklungsdiagnostischen Verfahren im engeren Sinne hinaus ausgedehnt werden auf die Institutionen, in denen die kindliche Entwicklung im weiteren Sinne, mit Blick auf ihre optimale Förderung, unter Beobachtung steht: die Erziehungs- und Bildungseinrichtungen sowie die Familie.

\section{Literatur}

Alanen, L. (2000) Visions of a social theory of childhood, Childhood. A global journal of child research, 7: 493-506.

American Academy of Pediatrics et al. (Hrsg.) (2006) 'Identifying infants and young children with developmental disorders in the medical home: an algorithm for developmental surveillance and screening', Pediatrics, 118 (4): 1808-1809.

Armstrong, D. (1983) Political Anatomy of the Body: Medical Knowledge in Britain in the Twentieth Century. Cambridge: Cambridge University Press.

Bayern: Bayrisches Landesamt für Gesundheit und Lebensmittelsicherheit (Hrsg.) (2006) Gesundheit der Vorschulkinder in Bayern. Ergebnisse zur Schuleingangsuntersuchung zum Schuljahr 2004/2005. Statistisch - epidemiologischer Bericht. Erlangen. http://www.kinderumweltgesundheit.de/KUG/index2/pdf/gbe/6099_1.pdf; zuletzt abgerufen am 16.01.2009.

Bayley, N. (2005) Bayley Scales of Infant and Toddler Development, Third Edition (Bayley-III). Oxford: Pearson Assessment.

Berlin: Senatsverwaltung für Gesundheit, Soziales und Verbraucherschutz (Hrsg.) (2006) Zur gesundheitlichen und sozialen Lage von Kindern in Berlin. Spezialbericht 2006-1. Berlin.

Bernfeld, S. (1967; orig. 1925) Sisyphos oder die Grenzen der Erziehung. Frankfurt a.M.: Suhrkamp.

Betz, T. (2008) Ungleiche Kindheiten. Theoretische und empirische Analysen zur Sozialberichterstattung über Kinder. Weinheim: Juventa.

Bollig, S. (2008) „Praktiken der Instrumentierung“. Methodologische und methodische Überlegungen zur ethnografischen Analyse materialer Dokumentationspraktiken in kinderärztlichen Vorsorgeuntersuchungen. Zeitschrift für Soziologie der Erziehung und Sozialisation, 28: 301-315.

Bollig, S. und M. Ott (2008) Entwicklung auf dem Prüfstand: zum praktischen Management von Normalität in Kindervorsorgeuntersuchungen, in $\mathrm{H}$. Kelle und A. Tervooren (Hrsg.) Ganz normale Kinder. Heterogenität und Standardisierung der kindlichen Entwicklung. Weinheim: Juventa, S. 207-224.

Bollig, S. und H. Kelle (2009) Früherkennung und Prävention von Entwicklungsstörungen: Medizinisierung und Pädagogisierung der frühen Kindheit aus praxisanalytischer Perspektive, in M. Behnisch und M. Winkler (Hrsg.) Soziale Arbeit und Naturwissenschaften. Positionen in sich verändernden Handlungsfeldern. München: Reinhardt.

Bühler-Niederberger, D. (1991) Legasthenie. Geschichte und Folgen einer Pathologisierung. Opladen: Leske+Budrich.

Bühler-Niederberger, D. (2005) Kindheit und die Ordnung der Verhältnisse. Von der Macht der Unschuld zum kreativen Individuum. Weinheim: Juventa

Burman, E. (1994) Deconstructing Developmental Psychology. London: Routledge.

Burman, E. (2007) Developments. Child, Image, Nation. London: Routledge.

Christensen, P. H. (2003) Kindheit und die kulturelle Konstitution verletzlicher Körper, in: H. Hengst und H. Kelle (Hrsg.) Kinder - Körper - Identitäten. Weinheim: Juventa, S. 115-136.

Conrad, P. (1976) The Discovery of Hyperkinesis: Notes on the Medicalization of Deviant Behavior. Social Problems, 23: 12-21.

Corsaro, W. A. (1997) The sociology of childhood. Thousand Oaks: Pine Forge Press.

Cravens, H. (1985) Child-Saving in the Age of Professionalism, 1915-1930, in: J. M. Hawes and R. N. Hyner (Hrsg.) American Childhood: a research guide and historical handbook. Westport, Conn.: Greenwood Press, S. 415-488.

Depaepe, M. (1993) Zum Wohl des Kindes? Pädologie, pädagogische Psychologie und experimentelle Pädagogik in Europa und den USA, 1890-1940. Weinheim: Deutscher Studien Verlag. 
Featherstone, M. und M. Hepworth (1998) Ageing, the lifecourse, and the sociology of embodiment, in: G. Scambler und P. Higgs (Hrsg.) Modernity, medicine, and health: medical sociology towards 2000. London: Routledge, S. 147-175.

Foucault, M. (2003) Die Anormalen: Vorlesungen am Collège de France (1974-1975). Frankfurt a.M.: Suhrkamp.

Frankenburg, W.K., J. Dodds, P. Archer, H. Shapiro and B. Bresnick (1992) 'The Denver II: a major revision and restandardization of the Denver Developmental Screening Test', Pediatrics, 89 (1): 91-7.

Fuhs, Burkhard (2003) Der Körper als Grenze zwischen den Generationen, in H. Hengst und H. Kelle (Hrsg.) Kinder, Körper, Identitäten. Weinheim: Juventa, S. 51-72.

Gerhard, U., J. Link und E. Schulte-Holtey (Hrsg.) (2001) Infografiken, Medien, Normalisierung. Zur Kartografie politisch-sozialer Landschaften. Heidelberg: Wissenschaftsverlag der Autoren.

Hasse, R. und G. Krücken (2005) Neo-Institutionalismus. Bielefeld: Transkript

Hendrick, H. (2003) Child Welfare: Historical Dimensions, Contemporary Debate. Bristol: The Policy Press.

Hengst, H. und H. Kelle (Hrsg.) (2003) Kinder, Körper, Identitäten. Weinheim: Juventa.

Hengst, H. und H. Zeiher (Hrsg.) (2005) Kindheit soziologisch. Wiesbaden: VS.

Hessen: Hessisches Sozialministerium (Hrsg.) (2006): Hessischer Kinder- und Jugendgesundheitsbericht. Wiesbaden.

http://www.hsm.hessen.de/iri/HSM Internet?cid=10f4c51a55f5412d1ec0b9d3292fd3b0; zuletzt abgerufen am 16.01.2009.

Hessen: Hessisches Sozialministerium/ Hessisches Kultusministerium (Hrsg.) (2007) Bildung von Anfang an. Bildungs- und Erziehungsplan für Kinder von 0 bis 10 Jahren in Hessen. Wiesbaden. http://www.kultusministerium.hessen.de/irj/HKM Internet?uid=422503e0-cf26-2901-be59-2697ccf4e69f (06-11-2008).

Hessischer Landtag (2007) Hessisches Gesetz zur Verbesserung des Gesundheitsschutzes für Kinder. Drucksache 16/8348.

Honig, M.-S. (1999) Entwurf einer Theorie der Kindheit. Frankfurt a.M.: Suhrkamp.

Hungerland, B. (2003) „Und so gedeiht das Baby!“ Altersgerechte Entwicklung und Gesundheit als gesellschaftliche Norm und Leistung, in $\mathrm{H}$. Hengst und $\mathrm{H}$. Kelle (Hrsg.): Kinder - Körper - Identitäten. Weinheim: Juventa, S. 139-160.

James, A., C. Jenks und A. Prout (1998) Theorizing childhood. Cambridge: Polity Press.

James, A. (2000) Embodied Being(s): Understanding the Self and the Body in Childhood, in A. Prout (Hrsg.): The Body, Childhood, and Society. Houndsmill: Macmillan Press, S. 19-37.

Joos, M. (2000) Die soziale Lage der Kinder. Sozialberichterstattung über die Lebensverhältnisse von Kindern in Deutschland. Weinheim: Juventa.

Kelle, H. (1997) „Wir und die Anderen“. Die interaktive Herstellung von Schulklassen durch Kinder, in S. Hirschauer und K. Amann (Hrsg.) Die Befremdung der eigenen Kultur. Zur ethnographischen Herausforderung soziologischer Empirie. Frankfurt: Suhrkamp, S. 138-167.

Kelle, H. (2001) The Discourse of "Development". How 9 to 12-Year-Old School Children construct 'Childish' and 'Further Developed' Identities within their Peer Culture, Childhood. A Global Journal of Child Research, 8: 95-114.

Kelle, H. (2005) Die Differenzierung der Generationen als kulturelle Praxis, in: H. Hengst und H. Zeiher (Hrsg.) Kindheit soziologisch. Wiesbaden: VS, S. 83-108.

Kelle, H. (2006) Sprachtests - ethnographisch betrachtet. Ein Beitrag zur Kulturanalyse frühdiagnostischer Testverfahren. Zeitschrift für qualitative Bildungs-, Beratungs-, und Sozialforschung (ZBBS), 7 (2): 271-291.

Kelle, H. (2007a) „Altersgemäße Entwicklung“ als Maßstab und Soll. Zur praktischen Anthropologie kindermedizinischer Vorsorgeuntersuchungen. 52. Beiheft der Zeitschrift für Pädagogik, S. 110-122.

Kelle, H. (2007b) „Ganz normal“: Die Repräsentation von Kinderkörpernormen in Somatogrammen. Eine praxisanalytische Exploration kinderärztlicher Vorsorgeinstrumente. Zeitschrift für Soziologie (ZfS), 36 (3): 197-216.

Kelle, H. und A. Tervooren (2008) Ganz normale Kinder. Heterogenität und Standardisierung der kindlichen Entwicklung. Weinheim: Juventa.

Kelle, H. (2008) „Normale“ kindliche Entwicklung als kulturelles und gesundheitspolitisches Projekt, in $\mathrm{H}$. Kelle und A. Tervooren (Hrsg.) Ganz normale Kinder. Heterogenität und Standardisierung der kindlichen Entwicklung. Weinheim: Juventa, S. 187-205. 
Kelle, H. und M. Ott (2009) Standardisierung der frühen kindlichen „Entwicklung“ und „Bildung“ in Kindervorsorgeuntersuchungen, erscheint in J. Bilstein und J. Ecarius (Hrsg.) Standardisierung Kanonisierung. Erziehungswissenschaftliche Reflexionen. Wiesbaden: VS

Klaus, A. (1993) Every Child a Lion. The Origins of Maternal and Infant Health Policy in the United States and France, 1890-1920. Ithaca: Cornell University Press.

Knorr Cetina, K. (2002) Wissenskulturen: ein Vergleich naturwissenschaftlicher Wissensformen. Frankfurt a.M.: Suhrkamp.

Kontopodis, M. (2007) Human Develoment as Semiotic-Material Ordering: Sketching a Relational Developmental Psychology, Critical Social Studies, 9: 5-20.

Kränzl-Nagl, R., J. Mierendorff und Th. Olk (Hrsg.) (2003) Kindheit im Wohlfahrtsstaat. Gesellschaftliche und politische Herausforderungen. Frankfurt: Campus.

Law, J. und A. Mol (2002) Complexities. Social Studies of Knowledge Practices. Durham: Duke University Press.

Leu, H. R. (Hrsg.) (2002) Sozialberichterstattung von Kindern. Opladen: Leske + Budrich.

Leu, H. R. (2008) Beobachtung in Bildungs- und Lernprozessen der frühpädagogischen Praxis, In W. Thole, H.-G. Rossbach, M. Fölling-Albers und R. Tippelt (Hrsg.) Bildung in der frühen Kindheit in Wissenschaft und Lehre. Opladen: Verlag Barbara Budrich, S.165-179.

Link, J. (2008) Zum diskursanalytischen Konzept des flexiblen Normalismus, in H. Kelle und A. Tervooren (Hrsg.) Ganz normale Kinder. Heterogenität und Standardisierung der kindlichen Entwicklung. Weinheim: Juventa, S. 59-72.

Lischeid, T. (2001) Vom „Schreibstammeln bei Halbidioten“ zur „umschriebenen LRS“. Diagnostische Kurvenprofile in der Legasthenieforschung, in: U. Gerhard, J. Link und E. Schulte-Holtey (Hrsg.) Infografiken, Medien, Normalisierung. Heidelberg: Wissenschaftsverlag der Autoren, S. 205-222.

Macha, T. und F. Petermann (2006) Entwicklungsdiagnostik, in F. Petermann und M. Eid (Hrsg.) Handbuch der Psychologischen Diagnostik. Göttingen: Hogrefe, S. 594-602.

Mayall, B. (1996) Children, Health, and the Social Order. Buckingham: Open University Press.

Nadesan, M.H. (2008) Governmentality, Biopower, and Everyday Life. London and New York: Routledge.

Olk, Th. (2007) Kinder im Sozialinvestitionsstaat, Zeitschrift für Soziologie der Erziehung und Sozialisation, 27: 43-57.

Place, B. (2000) Constructing the Bodies of III Children in the Intensive Care Unit, in: A. Prout (Hrsg.): The Body, Childhood, and Society. Houndsmill: Macmillan Press, S. 172-194.

Prout, A. (Hrsg.) (2000) The Body, Childhood, and Society. Houndsmill: Macmillan Press.

RKI: Robert Koch-Institut (Hrsg.) (2007) Ergebnisse des Kinder- und Jugendgesundheitssurveys (KiGGS). Bundesgesundheitsblatt. Bd. 50 (Heft 5/6)

Schmid, P. (2001) Vätertagebücher des ausgehenden 18. Jahrhunderts. Zu Anfängen der empirischen Erforschung von Säuglingen und Kleinkindern, in: I. Behnken und J. Zinnecker (Hrsg.): Kinder - Kindheit - Lebensgeschichte. Seelze-Velber: Kallmeyer, S. 325-339.

Smith, Dorothy E. (2002) Texts and the ontology of organizations and institutions' Studies in Cultures, Organizations and Societies, (Special Issue 'Studies in Institutional Ethnography') 7 (2): 159-198.

Tervooren, A. (2006) Im Spielraum von Geschlecht und Begehren. Ethnographie der ausgehenden Kindheit. Weinheim und München: Juventa.

Tervooren, A. (2008) „Auswickeln“, Entwickeln und Vergleichen. Kinder unter Beobachtung, in: Kelle, H. und A. Tervooren (Hrsg.) Ganz normale Kinder. Heterogenität und Standardisierung der kindlichen Entwicklung. Weinheim: Juventa 2008, S. 41-58.

Turmel, A. (1997) Childhood and Normalcy: Classification, Numerical Regularities, and Tabulations. International Journal of Educational Research, 27: 661-672.

Turmel, A. (2008) A Historical Sociology of Childhood. Developmental Thinking, Categorization and Graphic Visualization. Cambridge: Cambridge University Press.

Uprichard, E. (2008) Children as 'Beings and Becomings': Children, Childhood and Temporality. Children and Society, 22: 303-313.

Warming Nielsen, H. und J. Kampmann (2007) Children in Command of Time and Space? in: H. Zeiher et al. (Hrsg.) Flexible Childhood? Exploring Children's Welfare in Time and Space. Vol. 2 of COST A19: Children's Welfare. Odense: University Press of Southern Denmark, S. 191-214.

Wintersberger, H., L. Alanen, Th. Olk und J. Qvortrup (Hrsg.) (2007) Cildhood, Generational Order, and the Welfare State: Exploring Children's Social and Economic Welfare. Vol. 1 of COST A19: Children's Welfare. Odense: University Press of Southern Denmark. 
Woodhead, M. (1999) Reconstructing Developmental Psychology - Some First Steps. Children and Society, 13: 3-19.

Woodhead, M. (2009) Child Development and the Development of childhood, in J. Qvortrup, W.A. Corsaro und M.S. Honig (Hrsg.) Handbook of Childhood Studies. PalgraveMacmillan.

World Health Organization (Hrsg.) (1993) ICD-10. The ICD-10 Classification of Mental and Behavioral Disorders. Geneva: WHO.

World Health Organization (WHO) (2006) WHO Child Growth Standards: Length/Height-for-age, Weightfor-age, Weight-for-length, Weight-for-height and Body Mass Index-for-age. Methods and Development. Nonserial Publication WHO.

Zeiher, H. (1996) Kinder in der Gesellschaft und Kindheit in der Soziologie. Zeitschrift für Sozialisationsforschung und Erziehungssoziologie, 16: 26-46. 